This item was submitted to Loughborough's Research Repository by the author.

Items in Figshare are protected by copyright, with all rights reserved, unless otherwise indicated.

\title{
Magnetic correlations in paramagnetic iron
}

PLEASE CITE THE PUBLISHED VERSION

PUBLISHER

(C) Institute of Physics

LICENCE

CC BY-NC-ND 4.0

REPOSITORY RECORD

Samson, J.H.. 2019. "Magnetic Correlations in Paramagnetic Iron”. figshare. https://hdl.handle.net/2134/2232. 


\title{
MAGNETIC CORRELATIONS IN PARAMAGNETIC IRON
}

\section{J H SAMSON}

Department of Physics, Loughborough University of Technology, Loughborough, Leics, LE11 3TU England.

PACS. 75.10L - Band and itinerant models

PACS. 75.50B - Ferromagnetism of Fe and its alloys

PACS. 05.20 - Statistical mechanics

\begin{abstract}
A method is described for calculating the extent of short-range order (SRO) in the paramagnetic state of magnetic transition metals. An energy $V$ is calculated for a set of configurations of the exchange field. An entropy $S$ is attached to each configuration; this is the logarithm of the number of configurations with the same degree of long- and short-range magnetic order. The SRO is then found by minimizing the resulting free energy $V$-TS with respect to a near-neighbour correlation. The resulting Curie temperature $T_{\mathrm{c}}$ and the magnetic entropy are in good agreement with experiment. The corresponding nearest neighbour correlation is small (approximately $\cos 74^{\circ}$ ) at $T_{\mathrm{c}}$.
\end{abstract}

Published J Phys: Condens Matter 1 6717-6729 (1989) 


\section{Introduction}

The nature of magnetic correlations in the paramagnetic phase of the magnetic transition metals is still an open question. The ground state of $\mathrm{Fe}$ is well described by band theory and the transition by the disordering of local magnetic moments. Thermal properties such as specific heat, thermal expansion, spontaneous magnetovolume effect and susceptibility are compatible with a Heisenberg model with localised magnetic moments of magnitude close to the ground state value of $2.2 \mu_{\mathrm{B}}$. However, polarised neutron measurements (Johnson et al 1987) of the spinspin correlation function above the Curie temperature $T_{\mathrm{C}}$ show considerably less scattering at large wavevectors than predicted by the Heisenberg model. Korenman et al (1977) have interpreted the lack of weight at short wavelengths in terms of the existence of considerable magnetic short-range order (SRO) in the paramagnetic state. Edwards (1980) has criticised this interpretation as being incompatible with the thermal data, which show no evidence for unusual SRO in the paramagnetic state, and has explained the small magnitude of the moments in terms of missing weight at short wavelengths. The magnitude of the magnetic moments, $1.55 \mu_{\mathrm{B}}$ at $1.25 T_{\mathrm{C}}$, observed by integrating the scattering data, is considerably reduced from that in the ferromagnetic state, $2.2 \mu_{\mathrm{B}}$. This disagrees with the magnetovolume data, and would also imply a large increase in exchange energy (Kakehashi and Samson 1986). The distinction is one of time scales: the neutron measurements cover an energy window of approximately $2 k T_{\mathrm{C}}$. They therefore average over a time scale $\sim h / 2 k T_{\mathrm{C}}$ and do not see the faster fluctuations, which are mostly at short wavelengths. The fast fluctuations do however contribute to the thermal properties. Angle-resolved photoemission studies of the electronic structure of paramagnetic iron (Kisker et al 1985) are believed to average over an intermediate timescale. Haines et al (1985) and Clauberg et al (1985) have simulated these spectra by calculating the photoemission from random systems with varying degrees of SRO. By fitting the data at $T_{\mathrm{C}}$ they find a lower bound to the nearest-neighbour correlation coefficient of 0.4 , using a Gaussian correlation function. The trend suggested by these results is that faster measurements observe less SRO. The question addressed here is whether the itinerancy of the $d$-electrons leads to longer-range static (zero-time) correlations and smaller local moments than in a Heisenberg model. In this paper we develop a method of calculating the static SRO.

In previous treatments the extent of SRO has often been assumed from the outset. In local band theory (Korenman et al 1977), the magnetisation varies sufficiently slowly in space and time that it can support a local band structure. In the opposite limit, Hubbard(1979), Hasegawa(1980), Gyorffy et al (1985) and others picture local moments disordered on an atomic scale, and apply the coherent potential approximation. A more general approach, due to Moriya and Takahashi $(1978,1984)$, interpolates between these limits. Another approach is to fit calculated energies or observed spin wave dispersions to a model Hamiltonian whose statistical mechanics has been well studied. Shastry et al(1981) have used the exactly soluble spherical model (SM) of Berlin and Kac(1952) in this context. This does lead to a small amount of SRO in the paramagnetic state, although Johnson et al (1987) have criticised the model for failing to give a correct description of the dynamics. The present approach can be thought of as a generalised SM that can be fitted to the energies of arbitrary configurations of the magnetisation.

The method developed here is a general method of determining the SRO by appending a configurational entropy to energies calculated by various means. Figure 
1 in section 6 shows the principle behind the method, which is a quantitative development of the ideas of Heine and Joynt (1988). They argue that itinerant magnets disorder on a longer length scale than insulators, perhaps corresponding to the wavevector $\mathbf{q}_{\mathrm{c}}$ at which the spin waves enter the Stoner continuum. This scale can be found by comparing the energy and entropy of configurations of the magnetisation disordered on different length scales, minimizing the free energy with respect to the angle between nearest neighbours. There is some freedom of choice here in defining the degree of SRO: they use a domain-wall approximation that is valid only in the paramagnetic state and therefore obtain a first-order transition from a perfectly ordered state to a state with a finite domain size at $T_{\mathrm{C}}$. In the present work we develop a more systematic definition of the SRO. In both cases, the existence of SRO depends on the existence of a kink in the energy at some length scale, which determines the correlation length at $\mathrm{T}_{\mathrm{C}}$. We take an energy $V$ and a configurational entropy $S$ for spiral configurations with an angle $\mathbf{q} \cdot \mathbf{r}_{i j}$ between nearest neighbour moments. We define a $S R O$ parameter $c$, such as the nearest neighbour correlation

$$
c_{1}=\frac{1}{8} \sum_{j=\mathrm{NN} \text { of } i} \cos \mathbf{q} \cdot \mathbf{r}_{i j} .
$$

We then minimise the free energy $F(c)=V(c)-T S(c)$ to obtain the thermodynamic potentials and SRO parameter. The energy $V$ is an effective Hamiltonian for the magnetic excitations, which can be evaluated in terms of the total energy of a configuration of the magnetisation. It is in general a highly complicated function with long-range, nonquadratic and many-atom interactions. The entropy is the logarithm of the number of configurations consistent with the same degree of SRO; it can be evaluated exactly. Here we count the number of configurations with the same nearest-neighbour correlation $\left\langle\mathbf{S}_{i} \cdot \mathbf{S}_{j}\right\rangle$ as in the spin spirals, or more generally use an average of the correlation over a few shells.

The method can be thought of as an extension either of mean field theory (MFT) or of the spherical model (SM). In MFT one takes the energy $V$ to be a function of the total magnetisation $M$ only, and associates a combinatorial entropy $S$ with each value of $M$. This entropy is the logarithm of the number of microstates consistent with this magnetisation. One then minimises the free energy $F(M)=V(M)-T S(M)$ to obtain the thermodynamic potentials and order parameter. The approximation is exact if the energy is a function of the order parameter only, as is the case for infinite-range interactions. It is clearly unsatisfactory for the present purpose, where we are interested in the SRO. We therefore introduce a SRO parameter, such as the nearest-neighbour correlation, in addition to the order parameter, and calculate the corresponding energy and entropy. Minimizing the free energy with respect to both parameters will give the SRO in the paramagnetic state, provided that the energy depends only weakly on the remaining variables.

The SM consists of a spin Hamiltonian

$$
H=-\frac{1}{2} \sum_{i j} J_{i j} s_{i} s_{j}-\sum_{i} B s_{i},
$$

with the averaged constraint

$$
\sum_{i} s_{i}^{2}=N
$$


replacing the $N$ constraints on the magnitudes of each spin $s_{i}$. The exact free energy, magnetisation and correlation function are known (Berlin and Kac 1952). In the present method the Hamiltonian is an arbitrary function of the three summations in equations (1.2-3), fitted to the calculated energies. At any temperature the entropy and correlation function will be the same as in the corresponding SM at some temperature, but the temperature dependence of these quantities does differ. Moriya and Takahashi $(1978,1984)$ use a similar Hamiltonian, with the $J_{i j}$ in general functions of the other summations, but with the Hamiltonian restricted to a linear dependence on the correlation function. There is considerable numerical evidence for the importance of nonquadratic interactions (Hubbard 1979, Lin-Chung and Holden 1981).

In section 2 we discuss the effective Hamiltonian that is to be used. The entropy, calculated in section 3, may however be combined with any effective Hamiltonian. Section 4 covers the thermodynamics and section 5 the correlation functions. The results for iron, given in section 6, do not exhibit significant SRO. These results are discussed in section 7. A brief account of the method and some preliminary results have appeared elsewhere (Samson 1989).

\section{Energy}

The first stage is the choice of an effective Hamiltonian for the magnetic system. This Hamiltonian should describe the statistical mechanics, and therefore the static SRO, but not necessarily the dynamics. Various forms of Hamiltonian may then be used: spin models, such as a classical or quantum (spin-1) Heisenberg model or the SM (Shastry et al 1981), density functional energies (Gyorffy et al 1985), and, as is usually the case, the free energy of electrons in a field as derived by the functional integral method. The entropy derived in section 3 can be appended to any of these. We use the Hamiltonian obtained from the functional integral, as the approximations may be improved systematically (Samson 1984, 1986). We start from the Hubbard Hamiltonian, written in the form

$$
H=H_{\text {band }}-I \sum_{i} \mathbf{S}_{i} . \mathbf{S}_{i}+\frac{U}{4} \sum_{i} n_{i}^{2}
$$

where $\mathbf{S}_{i}$ and $n_{i}$ are the total $d$-electron spin and number operators on the site $i, H_{\text {band }}$ describes the bandstructure of non-interacting electrons in five $d$-bands or nine $s p d$ bands and $I$ is the Stoner parameter. The transition is driven by the magnetic degrees of freedom, which we represent by an exchange field $\left\{\boldsymbol{\Delta}_{i}\right\}$, a 3-component timeindependent vector on each site. The statistical mechanics of the Hubbard model is then the same as that of an effective Hamiltonian or potential $V\left(\left\{\boldsymbol{\Delta}_{i}\right\}\right)$. We call a set of $\left\{\boldsymbol{\Delta}_{i}\right\}$ a configuration. The partition function is an integral over all configurations:

$$
Z=\frac{\int \prod_{i} \mathrm{~d}^{3} \boldsymbol{\Delta}_{i} \exp \left(-\beta V\left(\left\{\boldsymbol{\Delta}_{i}\right\}\right)\right)}{\int \prod_{i} \mathrm{~d}^{3} \boldsymbol{\Delta}_{i} \exp \left(-\beta V_{0}\left(\left\{\boldsymbol{\Delta}_{i}\right\}\right)\right)}
$$

Here $\beta=1 / k T$ and $V_{0}$ is the potential of a "free" exchange field. So far this is a tautology: there is considerable freedom in the choice of $V$ and $V_{0}$ that will still keep the partition function exact; the effective Hamiltonian will in general be temperaturedependent. Such a Hamiltonian could be obtained in principle by applying the Hubbard-Stratonovich transformation to equation (2.1): the spin-spin interaction is 
replaced by an interaction of the spins with a vector exchange field and the chargecharge interaction is replaced by the interaction of the charge density with a Coulomb field. The Coulomb field and the finite-frequency parts of the exchange field are then integrated out. The present work however is restricted to the static approximation: if the one-electron Hamiltonian for electrons in the exchange field,

$$
H_{\mathrm{el}}=H_{\text {band }}-\sum_{i} \Delta_{i} \cdot \mathbf{S}_{i}
$$

has eigenvalues $E_{\mathrm{el}}$, the effective Hamiltonian is

$$
\begin{aligned}
& V=\sum_{E_{\mathrm{el}}<E_{\mathrm{F}}} E_{\mathrm{el}}+V_{0} \\
& V_{0}=\sum_{i} \frac{\Delta_{i}^{2}}{4 I} .
\end{aligned}
$$

Here the Fermi distribution has been replaced by a step function in equation (2.4) and the saddle-point approximation has been used for the charge fluctuations. Quantum corrections to the static approximation may be added (Samson 1984,1986); their possible effect will be touched upon later.

The quantity of interest is the spin-spin correlation function, which will not be calculated directly. It can be derived from the correlation function of the exchange field, which we shall be calculating directly, by the following relations:

$$
\begin{aligned}
& \left.<\mathbf{S}_{i}\right\rangle_{H}=\frac{\left\langle\Delta_{i}\right\rangle}{2 I} \\
& \left.<\mathrm{T} \int_{0}^{\beta} \mathrm{d} \tau_{1} \int_{0}^{\beta} \mathrm{d} \tau_{2} \mathbf{S}_{i}\left(\tau_{1}\right) \cdot \mathbf{S}_{j}\left(\tau_{2}\right)\right\rangle_{H}=\frac{1}{4 I^{2}}\left\langle\Delta_{i} \cdot \Delta_{j}\right\rangle-\frac{3 k T}{2 I} \delta_{i j}
\end{aligned}
$$

where $\mathrm{T}$ is the imaginary-time-ordering operator, with the imaginary-time dependence of an operator $A$ given by

$$
A(\tau)=e^{H \tau} A e^{-H \tau} .
$$

The averages $<>_{H}$ on the left hand sides of equations (2.6-7) are exact thermal averages for the Hubbard model. The spin-spin correlation in equation (2.7) is approximately that obtained by integrating the neutron scattering intensity over an energy window from 0 to $k T$. The averages $<>$ on the right hand side are thermal averages over the effective Hamiltonian $V$. Thus the calculated correlation functions of the exchange field relate to the measured scattering intensity.

The effective Hamiltonian in equation (2.4) is a very complicated function of the $\boldsymbol{\Delta}_{i}$ that cannot in general be fitted by a Heisenberg model, except within restricted classes of configurations. It may however be evaluated for any given configuration by electronic structure techniques such as the the recursion method (You and Heine, Holden and You 1982). It is the results for sample configurations that will be used as input for the next stage of the calculation.

We need a suitable sample of configurations that will be typical of the ferromagnetic and paramagnetic states with specified correlation functions. These can be either random configurations selected from a probability distribution (Haines 
1985) or regular configurations. The former were used to model photoemission from the paramagnetic state of iron (Haines et al (1985), Clauberg et al (1985)). We use the latter here, in the form of frozen spin waves (SW), conical configurations of the exchange field with all atoms equivalent. The exchange field

$$
\boldsymbol{\Delta}_{i}=\left(\Delta \sin \theta \cos \mathbf{Q} \cdot \mathbf{r}_{i}, \Delta \sin \theta \sin \mathbf{Q} \cdot \mathbf{r}_{i}, \Delta \cos \theta\right)
$$

is specified by three parameters: the amplitude $\Delta$, the order parameter $\cos \theta$, and the wavevector $\mathbf{Q}$. In the present work we concentrate on the paramagnetic state, which is modelled by SW with $\theta=\pi / 2$, so that the exchange field lies in the $x y$ plane. We call these configurations spin spirals.

To calculate the statistical mechanics based on the energies of a small number of configurations one must make an assumption regarding the remaining configurations. We therefore assume the energy of any configuration to be equal to that of the corresponding SW; a value of the parameters $\Delta, \theta$ and $\mathbf{Q}$ needs to be assigned to an arbitrary configuration. This requires a change of variables. A suitable parametrisation is

$$
\begin{aligned}
& \mathbf{x}_{1}=\frac{1}{N} \sum_{i} \Delta_{i} \\
& X_{2}=\frac{1}{N} \sum_{i} \Delta_{i}^{2} \\
& X_{3}=\frac{1}{N} \sum_{i j} \lambda_{i j} \Delta_{i} \cdot \Delta_{j}-X_{1}^{2} \text { where } \sum_{j} \lambda_{i j}=1
\end{aligned}
$$

describing the order parameter, the exchange field amplitude and a SRO parameter respectively, $N$ being the number of atoms. These are now defined for any configuration and, for the SW of equation (2.9), take the values

$$
\begin{aligned}
& \mathbf{X}_{1}=(0,0, \Delta \cos \theta) \\
& X_{2}=\Delta^{2}
\end{aligned}
$$

and

$$
\begin{aligned}
& X_{3}=\quad \Delta^{2} \sin ^{2} \theta \quad c \\
& \text { where } \\
& c=\sum_{j} \lambda_{i j} \cos \mathbf{Q} \cdot \mathbf{r}_{i j}
\end{aligned}
$$

is the SRO parameter. The energy is then the trial potential $V_{\mathrm{T}}(\Delta, \theta, \mathbf{Q})$. The presence of both long- and short-range order parameters in the energy takes account of the range of interaction. The energies can be fitted to nonquadratic nearestneighbour interactions or, following Shastry et al (1981), quadratic longer-range interactions. More generally, they can be fitted to an arbitrary function of $\Delta, \theta$ and $c$, with the $\lambda_{i j}$ suitably selected and possibly functions of temperature, $\Delta$ and $\theta$. Such a fit would however require data for a much wider range of configurations than the spin spirals considered here. The correlation function could also be fitted by more than one parameter, giving, say, the nearest- and next-nearest-neighbour correlations, at the expense of much greater algebraic complexity. Sasaki (1985) has derived such an expression for a molecular crystal Hamiltonian. 
The energies used here are those recently calculated by Luchini and Heine (1989) for Fe with spin spirals of wavevector $Q$ in the [100] and [110] directions, using a full $s p d$-bandstructure. The alternating tilt energies they report refer to the state below $T_{\mathrm{C}}$ and are not needed here. In a nearest-neighbour Heisenberg model, a plot of these energies against the nearest-neighbour correlation should lie on a straight line whose slope is given by the exchange interaction. The observed deviation from a straight line can arise both from longer-range interactions and multi-atom or nonquadratic interactions. In this paper, as an illustration of the method, we treat two limiting cases for the energy consistent with the [100] data:

I An arbitrary function of the nearest neighbour correlation only,

$$
V(c)=\sum_{n} a_{n} c_{1}^{n}
$$

where the SRO parameter $c$ is equal to the nearest neighbour correlation $c_{1}$.

II

A linear function of a longer-range correlation function,

$$
V(c)=J(1-c)
$$

where the SRO parameter $c$ in this case is a linear combination

$$
c=\sum_{n} \lambda_{n} c_{n}
$$

of $n$th neighbour correlations

$$
c_{n}=\frac{1}{z_{n}} \sum_{j \text { in }} \frac{<\Delta_{i} \cdot \Delta_{j}>}{\Delta^{2}} .
$$

Here $z_{n}$ is the coordination number of the $n$th shell. The coefficients $\lambda_{n}$ are the $\lambda_{i j}$ for the $n$th shell, normalised as in equation (2.12), so that in the ferromagnetic ground state $c=c_{n}=1$. Case II is equivalent to a Heisenberg or spherical model with exchange interactions $J \lambda_{n}$. If $\lambda_{i j}$ couples nearest neighbours only, spin spirals in the [100] direction on a BCC lattice of lattice constant $a$ correspond to

$$
c=\cos (Q a / 2), \quad \theta=\pi / 2 .
$$

Spin spirals in the [100] direction can be fitted to either of these forms. The parameters used are as follows, expressed in temperature units:

I: A least-squares cubic fit gives $a_{0}=1584 \mathrm{~K}, a_{1}=-1744 \mathrm{~K}, a_{2}=402 \mathrm{~K}$ and $a_{3}=-235 \mathrm{~K}$. II: $J=1376 \mathrm{~K}, J_{1} \sum \int J \lambda_{1}=225 \mathrm{~K}, J_{2} \int J \lambda_{2}=-101 \mathrm{~K}, J_{3} \int J \lambda_{3}=0, J_{4} \int J \lambda_{4}=7 \mathrm{~K}$ and $J_{5 . .} \int J \lambda_{5} \ldots=0$.

The fits in I and II give identical energies for spin spirals in the [100] direction. (In the results reported in section 4 a quintic rather than a cubic fit is used for case I.) With the spin spiral data used here there is little difference in the results between cases I and II, as the data do not deviate appreciably from a Heisenberg model.

\section{Entropy}


For each point in parameter space $\left(\mathbf{X}_{1}, X_{2}, X_{3}\right)$, or the equivalent $\mathrm{SW}(\Delta, \theta, c)$, we need to know the number of configurations in phase space $\left(\left\{\boldsymbol{\Delta}_{i}\right\}\right)$ satisfying the constraints (2.10-12). Each constraint corresponds to a $3 N-1$ dimensional surface in phase space, one point of which is the SW. The area of the intersection of those surfaces is the number of configurations compatible with the constraints, and the configurational entropy is the logarithm of that area. More generally, if these constraints are replaced by $n$ constraints

$$
X_{r}=f_{r}\left(\left\{\boldsymbol{\Delta}_{i}\right\}\right), 1 \leq r \leq n
$$

on macroscopic averages, with $n$ a small number of order 1 , the partition function of equation (2.2) can be written

$$
Z=\frac{\int \prod_{i} \mathrm{~d}^{3} \boldsymbol{\Delta}_{i} \exp \left(-\beta V\left(\left\{\boldsymbol{\Delta}_{i}\right\}\right)\right) \prod_{r=1}^{n} \int \mathrm{d} X_{r} \delta\left(X_{r}-f\left(\left\{\boldsymbol{\Delta}_{i}\right\}\right)\right.}{\int \prod_{i} \mathrm{~d}^{3} \boldsymbol{\Delta}_{i} \exp \left(-\beta V_{0}\left(\left\{\boldsymbol{\Delta}_{i}\right\}\right)\right)} .
$$

If the energy is a function of the parameters $\left\{X_{r}\right\}$ only, the integration over the exchange field can be carried out to leave

$$
Z=\frac{\int \prod_{r=1}^{n} \mathrm{~d} X_{r} \mathrm{e}^{-\beta\left(V_{\mathrm{T}}(X)-T S(X)\right)}}{Z_{0}}
$$

where $Z_{0}=\exp \left(-\beta F_{0}\right)$ is the denominator in equation (2.2) and the entropy is given by

$$
S(X)=k \ln \left[\int \prod_{i} \mathrm{~d}^{3} \boldsymbol{\Delta}_{i} \prod_{r=1}^{n} \delta\left(X_{r}-f_{r}\left(\left\{\boldsymbol{\Delta}_{i}\right\}\right)\right)\right] .
$$

This is the logarithm of the area of the intersection of $n$ surfaces in $N$ dimensions. The exponent in equation (3.3) is an extensive quantity, whilst the number of integrations is of order 1 . In the thermodynamic $(N \varnothing \bullet)$ limit the free energy is therefore given by the saddle point value

$$
F=\min _{X}\left[V_{\mathrm{T}^{-}} T S\right]-F_{0}
$$

and the values of the parameters by the position of the minimum.

We now return to specific results for the SW. The entropy can be evaluated exactly if the constraints are all linear or quadratic forms in the field. This is the case for the parametrisation of equations (2.10-12), where the surfaces are a plane, a sphere and a hyperboloid respectively. The derivation is in the appendix; the resulting entropy per atom is

$S(\Delta, \theta, c) / N=3 k \ln \Delta \sin \theta-3 k(\ln G(z)+\kappa(z)) / 2$,

where $G(z)$ is the lattice Green function

$$
G(z)=\frac{1}{\Omega_{\mathrm{BZ}}} \int_{\mathrm{BZ}} \mathrm{d}^{3} \mathbf{k} \frac{1}{z-\lambda(\mathbf{k})}
$$


and $\kappa(z)$ is its integral

$$
\kappa(z)=\frac{1}{\Omega_{\mathrm{BZ}}} \int_{\mathrm{BZ}} \mathrm{d}^{3} \mathbf{k} \ln (z-\lambda(\mathbf{k})) .
$$

The value of $z$ is determined implicitly in terms of the SRO parameter $c$ by

$$
c=z-1 / G(z)
$$

$\lambda(\mathbf{k})$ is the Fourier transform of $\lambda_{i j}$, normalised so that $\lambda(\mathbf{0})=1$. The first term in equation (3.6) corresponds to the amplitude of transverse fluctuations from the ferromagnetic state and the second term corresponds to a short-range-disorder entropy. In the ferromagnetic state $z=1 ; z$ increases with temperature in the paramagnetic state. The entropy therefore separates into three terms that depend only on the amplitude, order parameter and SRO respectively.

\section{Thermodynamic quantities}

We can calculate the exchange field amplitude, order parameter and SRO parameter for the trial Hamiltonian $V_{\mathrm{T}}$ by minimizing the free energy $V_{\mathrm{T}}-T S$ with respect to the parameters $\Delta, \theta$ and $c$. This gives

$$
\begin{aligned}
& \frac{3 k T}{\Delta}=\frac{\partial V_{\mathrm{T}}}{\partial \Delta} \\
& 3 k T \cot \theta=\frac{\partial V_{\mathrm{T}}}{\partial \theta}
\end{aligned}
$$

and

$$
\frac{3}{2} k T G(z)=\frac{-\partial V_{\mathrm{T}}}{\partial c}
$$

respectively. These equations can now be solved for the parameters.

Such a treatment in general requires a three-dimensional grid of data points. For the present work we make a further simplification. Here we are interested in the paramagnetic state, where $\theta=\pi / 2$. This leaves two free parameters, the amplitude $\Delta$ and the SRO parameter $c$. The spin spiral data used here (Luchini and Heine 1989) cover a one-dimensional slice through this space; they represent the "self-consistent" configurations in which $V_{\mathrm{T}}$ is minimized with respect to $\Delta$, which are those with moment $m(c)=\Delta / I$. This leads to a modified free energy, a function of $c$ only,

$$
F(c)=V_{\mathrm{T}}(c)-3 k T \ln m(c)+3 k T(\ln G(z)+\kappa(z)) / 2,
$$

in which amplitude fluctuations no longer appear. This is consistent with equations (4.1-3) if the longitudinal stiffness $\partial^{2} V_{\mathrm{T}} / \partial \Delta^{2}$ is independent of $c$. The temperature dependence of the SRO parameter is then given by

$$
\frac{3}{2} k T G(z)-3 k T \frac{d \ln m}{d c}=-\frac{d V_{\mathrm{T}}}{d c} .
$$

The Curie temperature is obtained by setting $z=1$ and using equation (3.9), giving 


$$
k T_{\mathrm{C}}=\frac{-2 \frac{d V_{\mathrm{T}}}{d c}}{3\left(G(1)-2 \frac{d \ln m}{d c}\right)}
$$

In the SM, $d V_{\mathrm{T}} / d c$ is simply $-J$, independent of $c$, and the second term in the denominator is absent. In the case of $\mathrm{Fe}$, where the moment is stable towards disordering, that term is small. The SRO parameter at and below $T_{\mathrm{C}}$ is

$$
c_{\max }=1-1 / G(1) .
$$

Below $T_{\mathrm{C}}$ this describes the correlation between transverse fluctuations. In case I, i.e. nearest-neighbour interactions (either quadratic or nonquadratic) on the BCC lattice, this is

$$
c_{\max }=0.282 \ldots=\cos \left(73.6 \ldots{ }^{\circ}\right) \quad .
$$

If the angle between nearest neighbours is constrained to be less than $73.6^{\circ}$, the system will order ferromagnetically. In case II, with longer range interactions, it is possible to obtain larger nearest neighbour correlations within the paramagnetic phase.

\section{Correlation functions and susceptibility}

The full correlation function can be determined once $c$ is known It is identical to that obtained from the SM with interactions proportional to $\lambda_{i j}$,

$$
\left\langle\left.\mathbf{\Delta}_{(\mathbf{q})}\right|^{2}\right\rangle=\frac{\Delta^{2}}{G(z)(z-\lambda(\mathbf{q}))}
$$

(Berlin and Kac 1952, Joyce 1972) where $z$ is given in terms of $c$ by equation (3.9). The temperature dependence of the correlation function depends on the temperature dependence of $c$ and therefore on the detailed form of the Hamiltonian. For example, if the slope of the energy increases rapidly with angle, or the self-consistent moment decreases rapidly with angle, the correlation length will decrease with temperature more slowly than in the SM.

The static susceptibility is given by

$$
\chi(\mathbf{q})=\frac{\mu_{0} \mu_{\mathrm{B}}^{2} g^{2} n}{12 k T I^{2}}\left(\left\langle\left.\mathbf{\Delta}_{\mathbf{\Delta}(\mathbf{q})}\right|^{2}\right\rangle-6 I k T\right) .
$$

Here $n$ is the number of atoms per unit volume and $g=2$. This can be derived from the functional integral. Writing $\Delta$ in terms of the self-consistent magnetisation gives

$$
\chi(\mathbf{q})=\frac{\mu_{0} \mu_{\mathrm{B}}^{2} g^{2} n}{12 k T}\left(\frac{m^{2}}{G(z)(z-\lambda(\mathbf{q}))}+O(k T / I)\right) .
$$

For the uniform susceptibility we substitute $\lambda(\mathbf{0})=1$. The $k T / I$ term, which contains a positive contribution from longitudinal fluctuations in $\Delta$ and a negative contribution from the second term in equation (5.2), is responsible for increasing the Curie constant from $S^{2}$ to $S(S+1)$ in the high-temperature limit. It will be ignored in the present treatment. 


\section{Results}

Figure 1 shows the energy $V_{\mathrm{T}}$, the scaled entropy -TS and the free energy $V_{\mathrm{T}}-T S$ as functions of the spin spiral angle at a temperature of $1.25 T_{\mathrm{C}}$ in case I, i.e. with an entropy calculated from a nearest-neighbour model. The position of the minimum gives the value of the SRO parameter; the minimum moves towards greater disorder with increasing temperature.

$\begin{array}{llll} & \text { I } & \text { II } & \text { experiment } \\ T_{\mathrm{C}} / \mathrm{K} & 820 & 670 & 1040 \\ \text { nn correlation }\left(T=T_{\mathrm{C}}\right) & 0.282=\cos 73.6^{\circ} & 0.254=\cos 75.3^{\circ} & >0.4 \mathrm{a} \\ \text { SRO parameter }\left(T=T_{\mathrm{C}}\right) & 0.282=\cos 73.6^{\circ} & 0.291=\cos 73.1^{\circ} & \\ <\mathbf{S}_{i \cdot} \mathbf{S}_{j}>_{\mathrm{nn}}\left(T=1.25 T_{\mathrm{C}}\right) & 0.232 & & 0.36 \mathrm{~b} \\ S(\infty)-S\left(T_{\mathrm{C}}\right) & 0.27 & 0.30 & 0.29 \mathrm{c}\end{array}$

a Haines et al (1985), Clauberg et al (1985) (photoemission)

b Ziebeck (unpublished) (neutron scattering)

c Bendick and Pepperhoff (1982) (heat capacity)

Table 1. Results for nearest-neighbour nonquadratic coupling (I) and longer-range quadratic coupling (II) compared with experimental data.

Table 1 compares calculated quantities with experimental values for both fits to the [100] spin spiral data. There is a slight difference between the two fits since the energies deviate a small amount from a nearest neighbour Heisenberg model. In both cases the SRO parameter is small at $T_{\mathrm{C}}$. The relevant experimental values are the nearest-neighbour correlation extracted from photoemission data and the nearest-neighbour spin-spin correlation $\left\langle\mathbf{S}_{i} . \mathbf{S}_{j}\right\rangle_{\mathrm{nn}}$ extracted from neutron data. For the latter, the table shows the calculated and experimental values of

$$
<\mathbf{S}_{i} \cdot \mathbf{S}_{j}>_{\mathrm{nn}}=c m^{2 / 4}
$$

where $m$ is the magnetisation, at a temperature of $1.25 T_{\mathrm{C}}$. Figure 2 shows the temperature dependence of the SRO parameter. Another measure of the SRO at $T_{\mathrm{C}}$ is the integrated entropy change between $T_{\mathrm{C}}$ and infinite temperatures. This is in good agreement with the experimental value, which has been extracted from Bendick and Pepperhoff's (1982) published plot of the magnetic heat capacity, although there is some uncertainty in separating a magnetic contribution from experimental data. The entropy is considerably less than the $k \ln (2 S+1)$ often quoted for the transition, indicating that most of the entropy enters below $T_{\mathrm{C}}$. (The integrated entropy change between 0 and $T_{\mathrm{C}}$ in this model would be infinite; this follows from the classical nature of the static approximation. The entropy expression of Heine and Joynt (1988), which assumes quantum spins, does give the value $k \ln (2 S+1)$ for the total entropy change.) Figure 3 shows the calculated heat capacity. Although the experimental data decrease much more rapidly than the calculated values, the entropy, as already mentioned, is in good agreement. Figure 4 shows the calculated susceptibility.

\section{Discussion}


The present calculation demonstrates that most of the static properties of iron in the paramagnetic state can be calculated by a method that takes the energies of configurations into account. This model does fail to describe the high-q part of the neutron scattering data (Johnson et al 1987), which shows much less weight (for energy transfers below $2 k T_{\mathrm{C}}$ ) than expected in this model. The reason for this is the failure of the static approximation correctly to describe excitations of energy greater than $k T$. At high $\mathbf{q}$ the spin waves have entered the Stoner continuum and there is very little scattering at low energies. To treat this one must go beyond the static approximation. One method would be to include quantum corrections in the effective Hamiltonian (which would then be temperature-dependent) (Samson 1984, 1986) and use the same technique for calculating the resulting statistical mechanics. If the corrections increase the Hamiltonian at large angles, the SRO will be enhanced. Another such effect, seen in calculations (unpublished) on a one-dimensional model and of possible relevance here, is that, as the amplitude increases with temperature, the transverse stiffness and therefore the effective exchange interactions increase. This also leads to larger SRO than in a Heisenberg model.

The method of attaching an area of phase space, and its corresponding entropy, to representative configurations can be applied to other forms of the effective Hamiltonian as well as to other statistical mechanical problems.

\section{Acknowledgments}

The author would like to thank M U Luchini for providing his data before publication, and both him and V Heine for useful discussions, and K R A Ziebeck for the neutron data and helpful discussions on their interpretation.

\section{Appendix}

The entropy equation (3.6) can be derived either through the exponential representation of the $\delta$-functions in equation (3.2) and the saddle point method or, more simply, by using known results of the SM (Berlin and Kac 1952). Consider the Hamiltonian

$$
H_{\mathrm{sp}}=-\frac{J}{2} \sum_{i j} \lambda_{l j} \Delta_{i} \cdot \Delta_{j}-\sum_{i} \mathbf{b} . \Delta_{i}
$$

with the spherical constraint

$$
\sum_{i} \Delta_{i}^{2}=N \Delta_{0}^{2}
$$

This Hamiltonian is a special case of the more general trial Hamiltonian $V_{\mathrm{T}}(\Delta, \theta, c)$ and can be written in a similar form:

$$
\frac{H_{\mathrm{sp}}}{N}=-\frac{1}{2} J \Delta^{2}\left(\cos ^{2} \theta+c \sin ^{2} \theta\right)+f(\Delta)-b \Delta \cos \theta
$$

where $f(\Delta)$ has an infinitely sharp minimum at $\Delta_{0}$. The entropy from equation (3.4), expressed in terms of the amplitude, energy and order parameter, becomes (up to a Jacobian that can be neglected in the thermodynamic limit) 


$$
\begin{aligned}
& S=k \ln \int \prod_{i} \mathrm{~d}^{3} \Delta_{i} \delta\left(\Delta^{2}-\frac{\sum_{i} \Delta_{i}^{2}}{N}\right) \delta\left(V_{\mathrm{T}}-H_{\mathrm{sp}}\left(\left\{\Delta_{i}\right\}\right)\right) \delta\left(\Delta \cos \theta-\frac{\sum_{i} \Delta_{i z}}{N}\right) \\
& =3 N k \ln \Delta+S_{\mathrm{sp}}\left(V_{\mathrm{T}}, \Delta \cos \theta\right) \quad,
\end{aligned}
$$

where $S_{\mathrm{sp}}$ is the entropy in the SM at a specified value of the energy and order parameter. This leads to the expression (3.6) for the entropy. (See e.g. equations 2.27, 2.15 and 2.22 of Joyce (1972), generalizing to a 3-component exchange field and neglecting additive constants)

The SRO parameter in the paramagnetic state is the ratio of the energy at temperature $T$ to that at $T=0$, as in equation (2.16). It is given by

$$
\begin{aligned}
c & =\frac{\int_{\mathrm{BZ}} d^{3} \mathbf{k} \frac{\lambda(\mathbf{k})}{z-\lambda(\mathbf{k})}}{\int_{\mathrm{BZ}} d^{3} \mathbf{k} \frac{1}{z-\lambda(\mathbf{k})}} \\
& =z-G(z) .
\end{aligned}
$$

In the case of nearest-neighbour coupling (case I) the analytic expression

$$
G(z)=\frac{4}{\pi^{2} z} K\left(\sqrt{\frac{1}{2}-\frac{1}{2} \sqrt{1-z^{-2}}}\right)
$$

(Morita and Horiguchi 1971) is used, where $K(k)$ is the complete elliptic integral of the first kind. 


\section{References}

Bendick W and Pepperhoff W 1982 Acta Metall 30 679-84

Berlin T H and Kac M 1952 Phys Rev 86 821-35

Clauberg R, Haines E M and Feder R 1985 Z Phys B62 31-41

Edwards D M 1980 J Magn Magn Mater 15-18 262-8

Gyorffy B L, Pindor A J, Staunton J, Stocks G M and Winter H 1985 J Phys F: Metal Phys $151337-$ 86

Haines E M 1985 J Comput Phys 60 353-75

Haines E M, Clauberg R and Feder R 1985 Phys Rev Lett 54 932-4

Hasegawa H 1980 J Phys Soc Japan 49 178-88, 963-71

Heine V and Joynt R 1988 Europhys Lett 5 81-5

Holden A J and You M V 1982 J Phys F (Metal Phys) 12 195-214

Hubbard J 1979 Phys Rev B19 2626-2636, B20 4584-4595

Johnson S M, Chaumet M, Neumann K-U, Thoma R and Ziebeck K R A 1987 J Magn Magn Mater 67 295-310

Joyce G S 1972 in Critical Phenomena vol 2, ed Domb C and Green M S, Academic (London) 375442

Kakehashi Y and Samson J H 1986 Phys Rev B34 1734-7

Kisker E, Schröder K, Gudat W and Campagna M 1985 Phys Rev B31 329-39

Korenman V, Murray J L and Prange R E 1977 Phys Rev B16 4032-47, 4048-57, 4058-62

Lin-Chung P J and Holden A J 1981 Phys Rev B23 3414-20

Luchini M U and Heine V 1989 to be published

Morita T and Horiguchi T 1971 J Math Phys 12 986-992

Moriya T and Takahashi Y 1978 J Phys Soc Japan 45 397-408, 1984 Annual Rev Materials Science 14 $1-25$

Samson J H 1984 Phys Rev B30 1437-47

Samson J H 1986 J Magn Magn Mater 54-57 983-4

Samson J H 1989 J Physique (Paris) Colloques in press (Proceedings of ICM '88)

Sasaki S 1985 J Phys C: Solid State Phys 18 101-26

Shastry B S, Edwards D M and Young A P 1981 J Phys C: Solid State Phys 14 L665-70

You M V and Heine V 1982 J Phys F (Metal Phys) 12 177-94

\section{Figure Captions}

1. Minimisation of the free energy as a function of nearest-neighbour angle. The thin line represents the energy, the broken line the scaled entropy and the thick line the free energy. The short-range order is found from the position of the minimum.

2. Short-range order, defined as the nearest-neighbour correlation function, in $\mathrm{Fe}$ as a function of temperature for case $\mathrm{I}$.

3. Heat capacity of $\mathrm{Fe}$ as a function of temperature for case I. The crosses are the data of Bendick and Pepperhoff (1982).

4. Inverse susceptibility of Fe as a function of temperature for case I. 


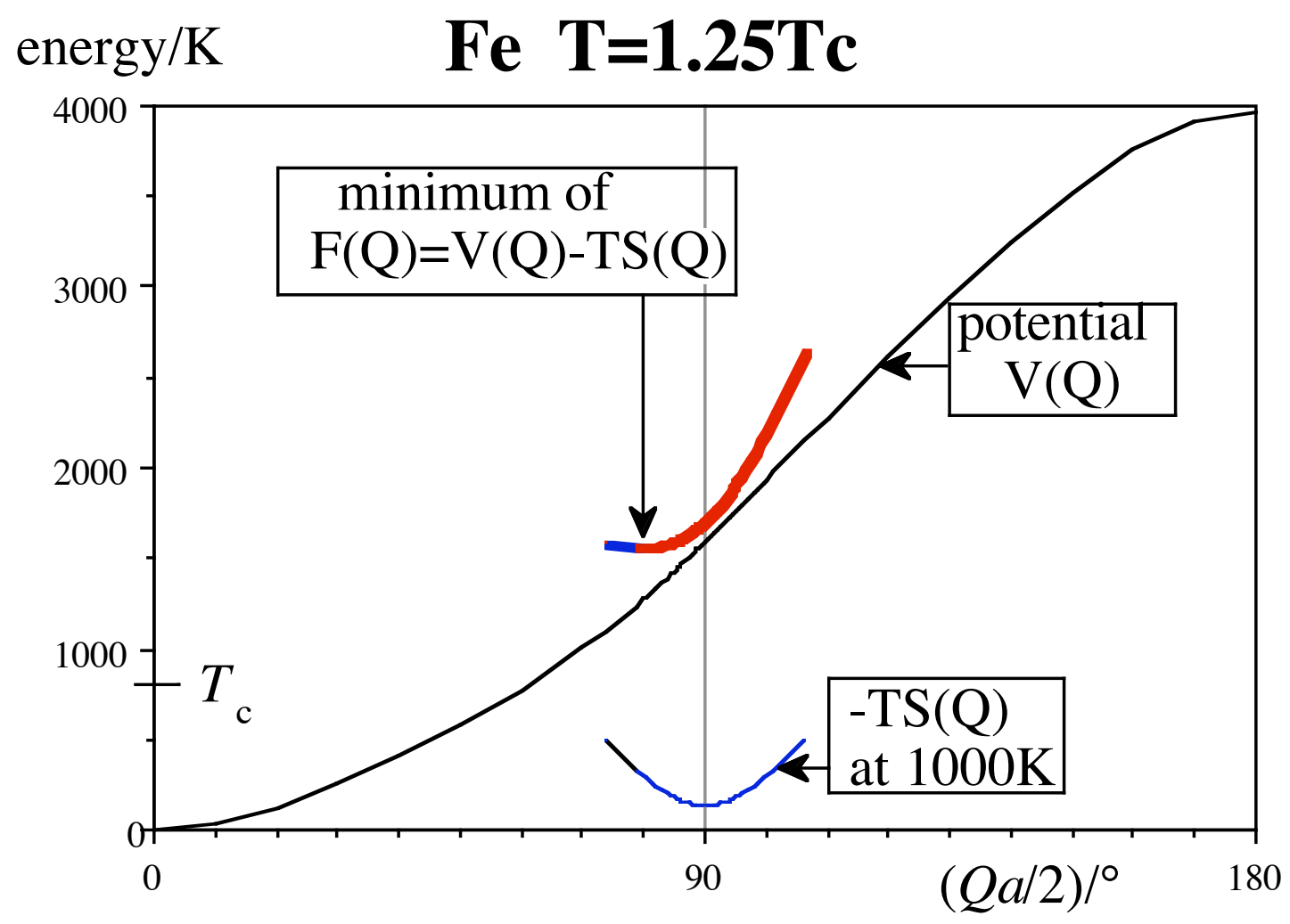

Figure 1 


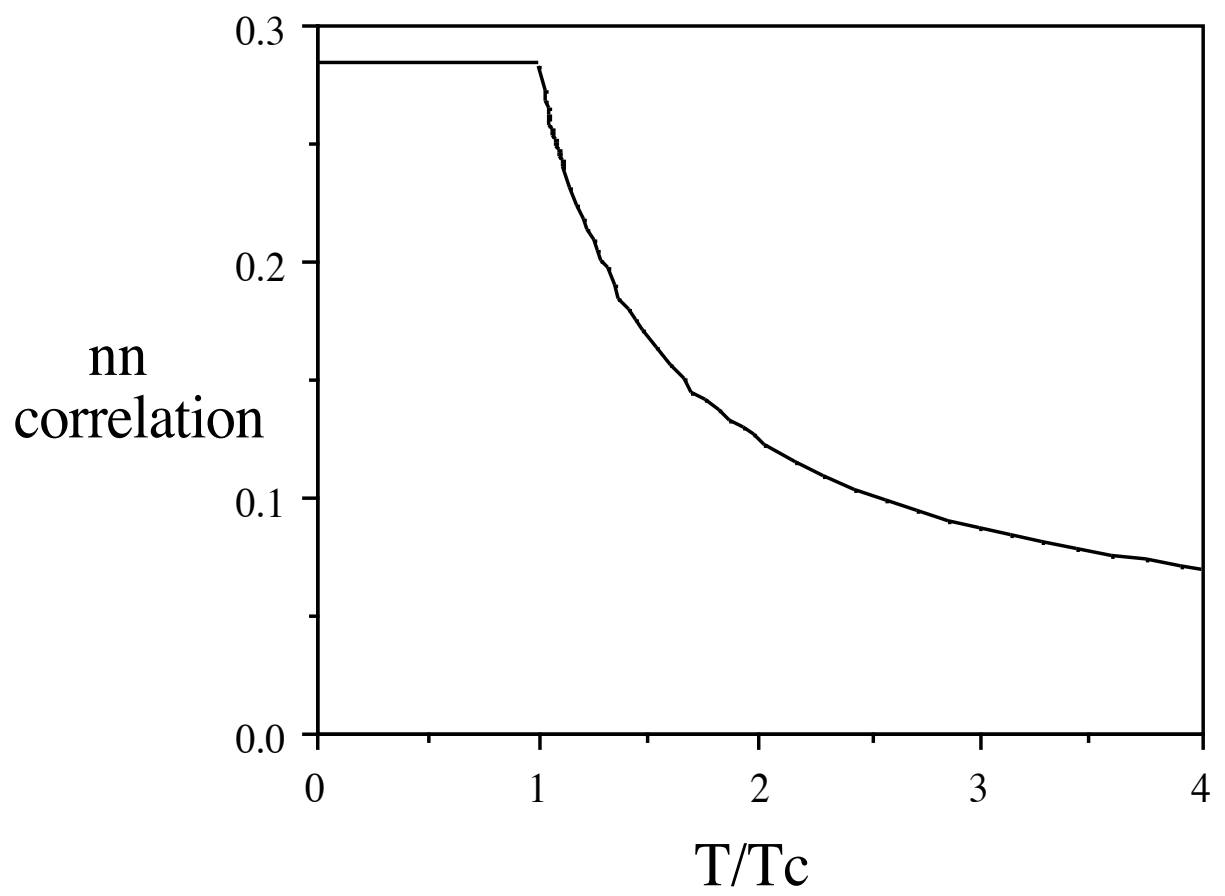

Figure 2 


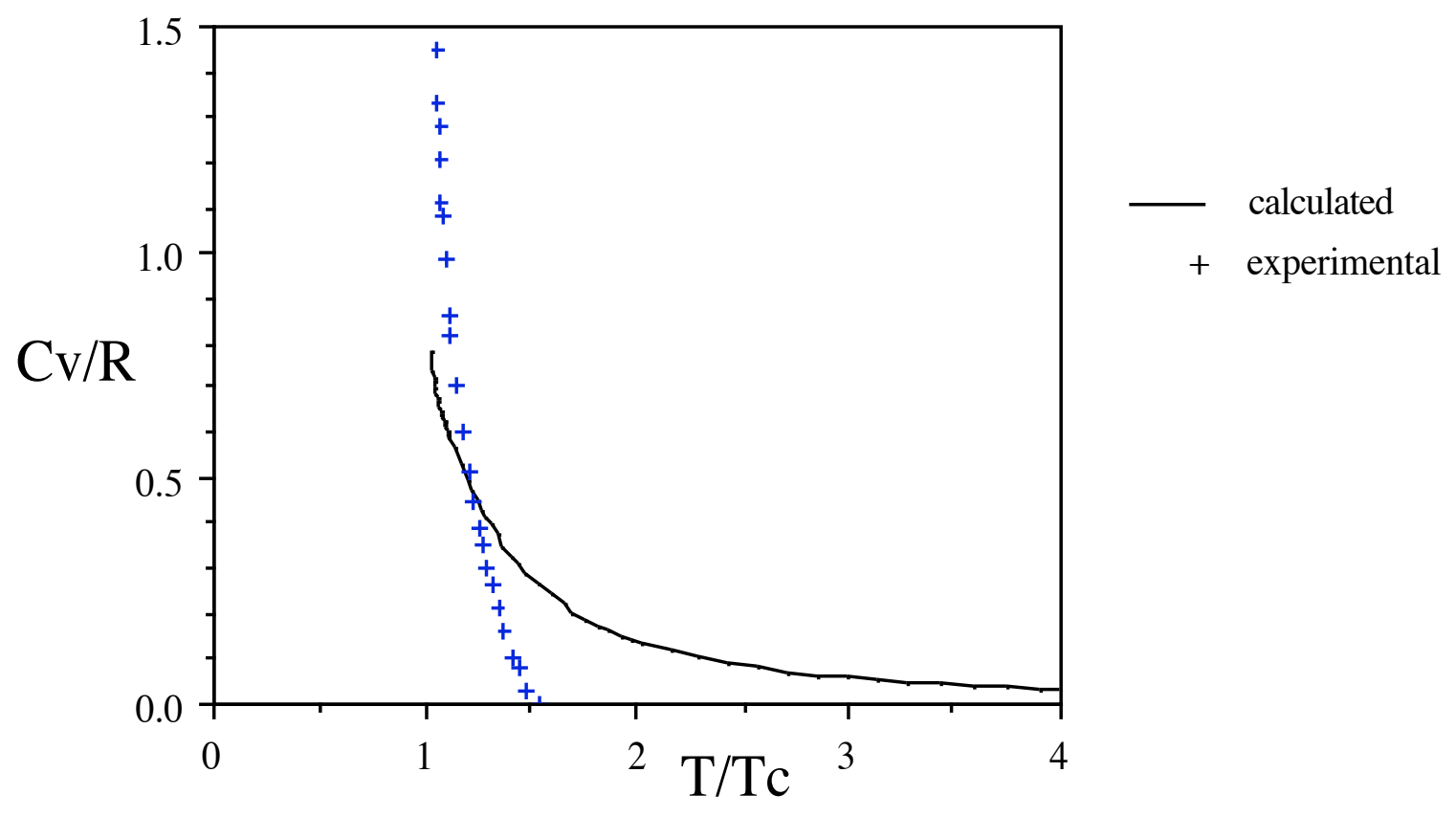

Figure 3 


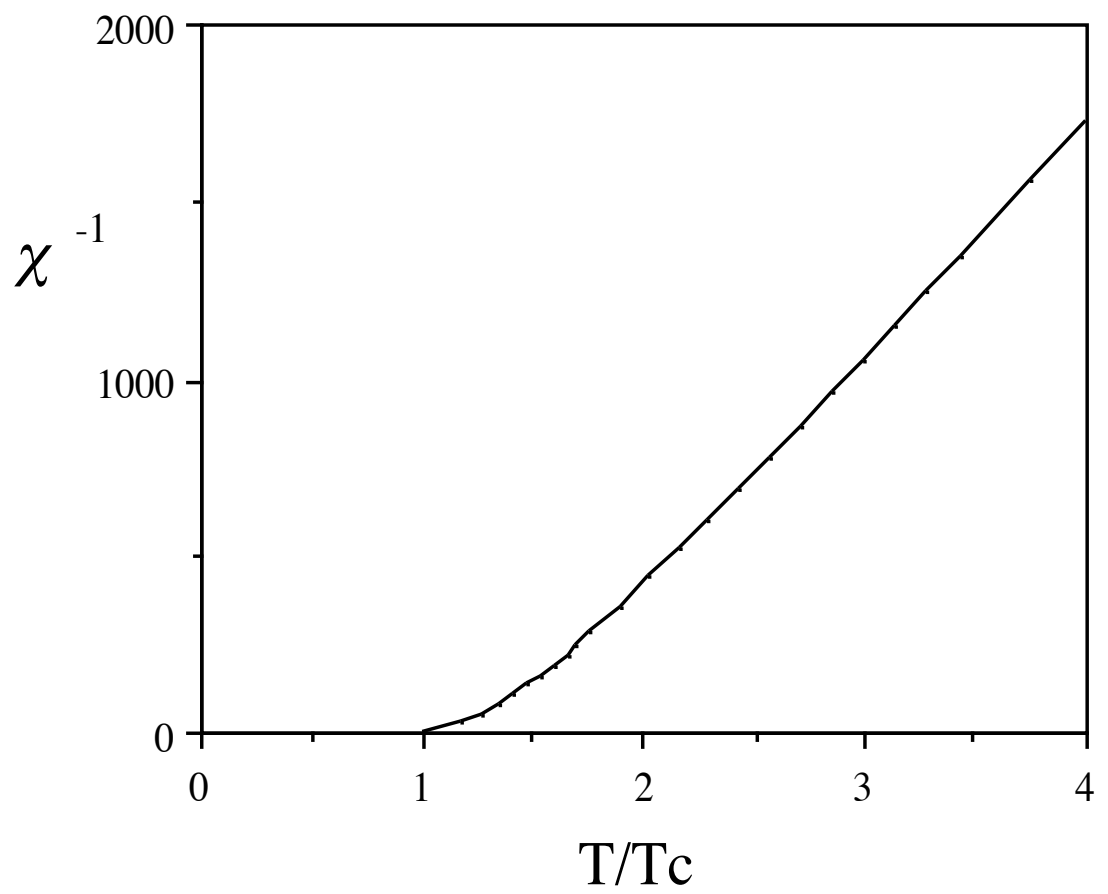

Figure 4 